\title{
¿Qué quejicosos! Buzón de quejas a través del aprendizaje cooperativo
}

\author{
ISABEL GARCÍA MARTÍNEZ \\ Universitat de València \\ igarmar8@alumni.uv.es
}

\begin{abstract}
Resumen: En este artículo se ofrece una experiencia práctica enmarcada dentro del aprendizaje cooperativo, que crea una interdependencia positiva propicia para el aprendizaje. Para ello, después de una breve aproximación teórica al aprendizaje cooperativo, la argumentación y los actos de habla de queja, así como del género textual «carta de reclamación», se expone la propuesta de nivel B2 organizada en diferentes pasos: la selección de equipos, el análisis de cartas de reclamación, la modificación de los equipos, la asignación de roles, así como la escritura de cartas y posterior evaluación. Se trabajan distintas destrezas, además de conseguir que los alumnos interactúen como en la vida real.
\end{abstract}

Palabras clave: aprendizaje cooperativo, carta de reclamación, argumentación, expresión escrita.

\begin{abstract}
This article offers a practical experience framed within cooperative learning, which creates a positive interdependence conducive to learning. To do so, after a brief theoretical approach to cooperative learning, argumentation and acts of speech of complaint, as well as the textual genre "letter of complaint", the proposal of level B2 organized in different steps: the selection of equipment, the analysis of complaint letters, the modification of the teams, the assignment of roles, as well as the writing of letters and subsequent evaluation. Different skills are worked on, as well as getting students to interact as in real life.
\end{abstract}

Keywords: cooperative learning, complaint letters, argumentation, writing skills. 


\section{Introducción}

Este artículo pretende ofrecer al docente una propuesta didáctica para llevar al aula la argumentación a partir del aprendizaje cooperativo. Surge de la necesidad de incluir los mecanismos argumentativos y el acto de la queja en las cartas de argumentación, que están presentes en el nivel B2 del MCER, en el proceso de enseñanza-aprendizaje del alumnado de E/LE. Por ello, comenzaremos por una breve aproximación al concepto de aprendizaje cooperativo para posteriormente introducirnos en las quejas como acto de habla dentro del género escrito.

El aprendizaje cooperativo (AC) o aprendizaje en cooperación se enmarca en la línea teórica del constructivismo, que surgió en EE.UU. a principios del siglo XX y que considera que los alumnos son los protagonistas de su proceso de enseñanzaaprendizaje, mientras que el profesor mantiene un papel no de mero transmisor de conocimientos, sino de facilitador y organizador del aprendizaje grupal.

Muchos son los autores que han contribuido a teorizar sobre este tipo de aprendizaje, algunos de los exponentes son Johnson y Johnson (1999: 5) quienes oponen el aprendizaje competitivo al aprendizaje cooperativo y a este lo definen como «el empleo didáctico de grupos reducidos en los que los alumnos trabajan juntos para maximizar su propio aprendizaje y el de los demás». Por su parte, Kagan (1994: 2-10) lo define como «una serie de estrategias instruccionales que incluyen a la interacción colaborativa de estudiante a estudiante, sobre algún tema, como una parte integral del proceso de aprendizaje». Aquí, en España, Cassany (2004: 16), uno de los principales autores que introduce el $\mathrm{AC}$ en $\mathrm{E} / \mathrm{LE}$, distingue entre equipo y grupo. «Los miembros del equipo han sido entrenados durante un cierto periodo para aprender conjuntamente, mientras que el grupo son solo aprendices sin formación ni conocimiento mutuo que se reúnen ocasionalmente para resolver un ejercicio».

En definitiva, podemos afirmar siguiendo a Ovejero (1990: 57), que «todo aprendizaje cooperativo es aprendizaje en grupo, pero no todo aprendizaje en grupo es aprendizaje cooperativo».

Alfageme González, M. B. (2003: 51) ha adaptado la propuesta de diferencias de grupos de aprendizaje cooperativo y de grupos de aprendizaje tradicional de Johnson, Johnson y Smith (1991: 25) en la siguiente tabla:

\begin{tabular}{|c|c|}
\hline Grupos de aprendizaje cooperativo & Grupos de aprendizaje tradicional \\
\hline Interdependencia positiva & Ninguna interdependencia \\
\hline Responsabilidad individual & Ninguna responsabilidad individual \\
\hline Grupo con miembros heterogéneos & Grupo con miembros homogéneos \\
\hline Dirección compartida & Dirección de un líder \\
\hline $\begin{array}{c}\text { El sujeto es responsable de lo que hacen los demás } \\
\text { miembros del grupo }\end{array}$ & $\begin{array}{l}\text { El sujeto solo es responsable de lo que hace él } \\
\text { mismo }\end{array}$ \\
\hline Se acentúa o enfatiza tanto la tarea como su & Se acentúa o enfatiza solo la tarea \\
\hline Se enseñan directamente habilidades sociales a los & $\begin{array}{l}\text { seciales, o se ignora si las poseen o no } \\
\text { somes }\end{array}$ \\
\hline sujetos & El profesor no hace caso del grupo \\
\hline $\begin{array}{c}\text { El profesor observa e interviene en el grupo } \\
\text { Suceden procesos en el grupo }\end{array}$ & Ningún proceso de grupo \\
\hline
\end{tabular}

Tabla 1. Grupos de aprendizaje cooperativo y aprendizaje tradicional.

El AC, según Johnson y Johnson (1999), propicia los siguientes beneficios en el 
alumnado: mayores esfuerzos por lograr un buen desempeño, relaciones más positivas entre los alumnos y una mayor salud mental. Además, estos autores afirman que para que funcione se debe tener en cuenta: la interdependencia positiva, la responsabilidad individual y grupal, la interacción estimuladora, las técnicas interpersonales y de equipo, así como la evaluación grupal.

En cuanto al docente, Johnson y Johnson (1999), el AC ayuda al docente a:

- Elevar el rendimiento de todos sus alumnos, incluidos los que tienen dificultades.

- Establecer relaciones positivas entre los alumnos, sentando así las bases de una comunidad de aprendizaje en la que se valore la diversidad.

- Proporcionar a los alumnos las experiencias que necesitan para lograr un saludable desarrollo social, psicológico y cognitivo.

\section{Las quejas como acto de habla en las cartas de reclamación}

Las cartas de reclamación, y como consecuencia las quejas, pertenecen a un género textual escrito muy dificultoso y de gran utilidad social y práctica. Siguiendo a Trosborg (1995:311), una de las estudiosas de este ámbito, consideramos queja «an illocutionary act in which the speaker expresses his/her disapproval, negative feelings etc. towards the state of affairs described in the proposition and for which he/she holds the hearer responsible, either directly or indirectly».

Por su parte, el MCER establece que el nivel B1 el aprendiente sabe plantear una queja o hacer una reclamación en su apartado de «interactuar para obtener bienes y servicios». En cambio, en el nivel B2, el aprendiente:

Tiene capacidad lingüística para negociar la solución de conflictos, como pueden ser reclamar una multa de tráfico injusta, pedir responsabilidad económica por daños causados en un piso o por culpabilidad en un accidente. Puede desarrollar su argumentación en caso de daños y perjuicios, utilizando un lenguaje persuasivo para reclamar una indemnización, y establece con claridad los límites de cualquier concesión que esté dispuesto a realizar

(Consejo de Europa, 2002: 79)

El Plan Curricular del Instituto Cervantes (PCIC) abarca las cartas de reclamación en los géneros de transmisión escrita en todos sus niveles. Como género de transmisión oral en el nivel $\mathrm{B} 2$ y $\mathrm{C} 1$ incluye la recepción y la producción de reclamaciones rutinarias en conversaciones transaccionales telefónicas de extensión media. En cambio, en el nivel C2 lo introduce en conversaciones transaccionales cara a cara. Además, en la descripción general del nivel B2 dice que los alumnos que alcanzan este nivel «disponen de capacidad lingüística suficiente como para plantear los detalles de un problema, presentar reclamaciones y resolver situaciones conflictivas recurriendo a su capacidad de argumentar y a un lenguaje persuasivo» (Instituto Cervantes, 2006: 20).

\section{Perfil de la actividad:}

Esta propuesta didáctica tiene como objetivos:

- Analizar y producir cartas de reclamación.

- El uso de las estrategias argumentativas.

- El desarrollo de las estrategias pragmáticas para formular el acto de la queja. 
Se puede llevar al aula a partir de un nivel B2.

Las competencias de la lengua que se trabajan son las siguientes:

- Dar una opinión argumentando a favor o en contra a través del medio escrito.

- Expresar acuerdo, acuerdo parcial y desacuerdo.

- Escribir una carta de reclamación.

Las destrezas presentes en la actividad son la interacción oral, la expresión y la comprensión escritas.

Se puede desarrollar en dos sesiones de 60 minutos.

\section{Proceso de la actividad}

\subsection{Selección de grupos}

Se trabaja en una clase de 9 alumnos, aunque puede ser modificable. Los grupos constan de 3 integrantes, pues es el número que consideramos idóneo para que se mantenga la intimidad y los participantes se sientan cómodos interaccionando. Además con este número se propicia una participación activa y responsable de todos los estudiantes y asimismo conseguimos diagnosticar más rápidamente las dificultades o conflictos que puedan surgir.

En cuanto a la distribución, Johnson y Johnson (1999: 17) dicen que «la productividad de un grupo está determinada por la capacidad de sus miembros para trabajar en equipo», por lo tanto, prestamos especial atención a este aspecto. Optamos por grupos heterogéneos que aseguran un amplio abanico de respuestas, rendimientos, puntos de vista, intereses y aportaciones. Siguiendo a los anteriores autores:

Los grupos heterogéneos tienden a promover un pensamiento más profundo, un mayor intercambio de explicaciones y una mayor tendencia a asumir puntos de vista durante los análisis de material, todo lo cual incrementa la comprensión, el razonamiento y la retención a largo plazo de los alumnos

(Johnson y Johnson, 1999: 18)

Esta distribución se hace al azar, se trata de un método fácil y eficaz que asegura la total heterogeneidad. Al tener 9 alumnos y querer hacer grupos de 3, enumeramos a cada estudiante con un número del 1 al 3 y después deben agruparse los alumnos que tengan el mismo número.

Cada grupo se sientan en círculo y de manera cercana, así pueden verse las caras, hablar sin molestar al resto de grupos y compartir los materiales que se les entrega. El docente debe tener suficiente espacio para poder circular por entre los grupos.

\subsection{Reparto de cartas de reclamación a cada grupo}

Después de formar los equipos, el docente entrega a cada uno de ellos una carta, todas con una temática diferente. En nuestro caso, serán tres quejas que responden a los siguientes conflictos:

-Se alquiló un apartamento en Málaga a 5 minutos de la playa a través de una página web y al llegar no cumplía ninguna característica de las que anunciaba. 
-Se tuvo un problema con el teléfono móvil y la compañía de telefonía no prestó ayuda.

-Un vuelo se ha cancelado y como consecuencia se han extraviado las maletas.

En el anexo se pueden consultar estas cartas.

\subsection{Análisis de las cartas de reclamación}

A los estudiantes se les proporcionan las anteriores cartas, una por equipo. Cada componente deberá llevar a cabo una reflexión individual y después deberán compartir sus creencias y pensamientos con el resto de miembros. Es importante que el profesor explique claramente el procedimiento y los objetivos de la actividad, incluyendo la forma de trabajar en grupo. Para este análisis, el docente proporcionará el siguiente material:

Reflexiona y subraya en el texto:

- ¿Cuál es la introducción? Recuerda que se trata de la contextualización del tema y la anticipación del desarrollo del escrito.

- ¿Cuál es la cuestión polémica?

- ¿Y el desarrollo?

- ¿Dónde está la opinión o la tesis?

- ¿Hay regla general?

- ¿Qué argumentos hay? ¿Qué recursos trabajados en clase se pueden observar? [verbos creer, pensar, opinar, locuciones: en mi opinión, desde mi punto de vista..., verbos como aceptar, temer(se), reconocer, admitir, sentir, preguntar(se)]

- ¿Citan alguna fuente?

- ¿Existe alguna contraargumentación? ¿Qué tipo de recursos encuentras? [Expresiones de acuerdo parcial: aunque, a pesar de que, hay que recordar que, no está claro que, es evidente, es un hecho, está claro que + indicativo, pero, sin embargo.

- ¿Cuál es la conclusión?

- ¿Qué fórmulas de cortesía encuentras en la carta?

- ¿Cuál es la fórmula de inicio? ¿Y la de cierre?

- ¿Hay uso de conectores para dar coherencia y cohesión al texto? Si es así, ¿cuáles? ¿De qué tipo son?

- ¿Hay algún tipo de estrategia de impersonalización?

En sesiones previas ya se han trabajado estos aspectos en el aula.

Mientras los equipos trabajan con el texto y su análisis, el docente debe controlar la práctica y guiar a los equipos que tengan alguna dificultad. Durante su circulación por la clase deberá asegurarse que todos los equipos han logrado la respuesta correcta.

\subsection{Modificación de los grupos}

Siguiendo a Aronson (1978) y su técnica Jigsaw, también conocida como Puzle o Rompecabezas, los estudiantes tras el análisis grupal se convierten en expertos de su texto, en este caso, de su carta de reclamación. Cambiarán el agrupamiento y los nuevos equipos estarán formados por un experto de cada carta. Ahora, por orden, es el momento de explicar y enseñar lo aprendido en la fase anterior al resto de compañeros, siguiendo para esto el guión de preguntas. 


\subsection{Asignación de roles}

A cada integrante del equipo se le asigna un rol, de esta manera se asegura la participación de todos y se comparte la responsabilidad en el resultado final. Estos roles son los siguientes: un encargado de la supervisión de turnos, otro encargado de sintetizar o compendiar las ideas y/o decisiones $\mathrm{y}$, por último, otro encargado de materializarlas, es decir, de escribirlas.

Cada equipo desempeña un papel que se les asigna para la preparación de la tarea posterior. Estos papeles son:

1. Joven que ha sufrido un accidente de coche y debe informar y realizar una reclamación a la compañía aseguradora.

2. Conductor que ha recibido una multa de tráfico injusta y debe reclamarla.

3. Estudiante universitario que vive en una calle con obras y debe pedir su fin.

Después los equipos deberán pensar, interactuar, argumentar y llegar a un acuerdo para poder escribir la carta eficazmente. Para esto el docente proporciona una serie de preguntas a los equipos:

1.

- ¿Cómo ha sido el accidente?

- ¿Dónde ha sido el accidente?

- ¿Qué daños he sufrido?

- ¿Cómo fueron los hechos?

- ¿La compañia aseguradora ha respondido?

- ¿Qué tono vamos a adoptar en la carta?

- ¿Qué recursos vamos a utilizar?

2.

- ¿Qué infracción vial se ha producido?

- ¿Por qué es injusta?

- ¿Cómo fueron los hechos?

- ¿Qué argumentos se van a utilizar?

- ¿Se pueden utilizar testimonios de testigos?

- ¿Qué tono vamos a adoptar en la carta?

- ¿Qué recursos vamos a utilizar?

3.

- ¿Cuál es el problema?

- ¿Por qué es un problema?

- ¿Cuánto tiempo hace que se vive este problema?

- ¿Se propone alguna solución?

- ¿Se ha informado a alguien? ¿Ha respondido?

- ¿Qué tono vamos a adoptar en la carta?

- ¿Qué recursos vamos a utilizar?

El docente durante este proceso deberá prestar atención a que todos los estudiantes estén participando y respetando las directrices que se han establecido.

\subsection{Escritura de cartas}

En esta fase los alumnos, por equipos, deben escribir sus propias cartas de reclamación 
siguiendo los modelos que han analizado previamente. Estos son los objetivos de las mismas:

- Informar de un accidente y realizar una reclamación a la compañía de seguros

- Reclamar una multa de tráfico injusta

- Pedir responsabilidad económica por daños causados en un piso

Después el docente las revisa y las corrige para la próxima sesión.

\subsection{Departamento de Atención al Cliente}

En esta nueva sesión, los equipos son Departamentos de Atención al Cliente, uno será una compañía aseguradora, otro la Dirección General de Tráfico y, por último, concejal de obras de un ayuntamiento. El docente reparte las cartas de la sesión anterior, cada equipo recibe una carta diferente a la que ha escrito. El objetivo es contestar al cliente insatisfecho, al ciudadano que se queja, redactando una nueva carta. Los estudiantes ya tienen una experiencia anterior y pueden seguir el mismo procedimiento.

\subsection{Evaluación}

Los equipos deberán hacer una reflexión y las cartas de los otros equipos a través de rúbricas. Las cartas se intercambian en cada equipo y cada equipo completa la siguiente tabla con referencia a la carta que le ha asignado el docente.

\begin{tabular}{|c|c|c|c|c|}
\hline Evaluación de la carta de reclamación & Insuficiente & Aceptable & Bien & Excelente \\
\hline \multicolumn{5}{|l|}{ La carta tiene una estructura adecuada y diferenciada } \\
\hline \multicolumn{5}{|l|}{ Los argumentos se adaptan a la situación } \\
\hline \multicolumn{5}{|l|}{ Cohesión y coherencia } \\
\hline \multicolumn{5}{|l|}{ Corrección y presentación } \\
\hline $\begin{array}{l}\text { Presenta todos los elementos que se han analizado } \\
\text { previamente }\end{array}$ & & & & \\
\hline Consecución de los objetivos propuestos & & & & \\
\hline
\end{tabular}

Tabla 2. Ficha de evaluación.

Asimismo cada estudiante reflexiona sobre su proceso de enseñanza-aprendizaje a través de esta autoevaluación.

\begin{tabular}{|c|c|c|c|c|}
\hline Autoevaluación & Insuficiente & Aceptable & Bien & Excelente \\
\hline \multicolumn{5}{|l|}{ Se ha valorado mi esfuerzo } \\
\hline $\begin{array}{l}\text { He aprendido de los materiales } \\
\text { procedimientos }\end{array}$ & $\mathrm{y}$ & & & \\
\hline \multicolumn{5}{|l|}{ Me he sentido cómodo trabajando en el equipo } \\
\hline \multicolumn{5}{|l|}{ He contribuido al equipo } \\
\hline He sido respetado como miembro & & & & \\
\hline
\end{tabular}

Tabla 3. Ficha de autoevaluación. 


\section{Conclusiones}

Este trabajo ha pretendido llevar al aula la cooperación como estrategia de aprendizaje para lograr analizar y escribir cartas de reclamación, consiguiendo un aprendizaje autónomo en el aula de español como lengua extranjera, en este caso de nivel B2. Se trabaja la queja como acto de habla, poniendo especial énfasis en la construcción de la argumentación en equipo, ya que es algo que presenta dificultades, tanto a nivel nativo como extranjero. Esta experiencia práctica consigue motivar a los estudiantes y conseguir una participación equitativa de todos los grupos, al mismo tiempo que han desarrollado estrategias pragmáticas para formular el acto de la queja, han opinado argumentando a favor o en contra $\mathrm{y}$ han expresado acuerdo, acuerdo parcial y desacuerdo. En definitiva, con esta actividad se trabajan distintas destrezas, además de conseguir que los alumnos interactúen como en la vida real.

\section{Bibliografía}

Aronson, E. (1978). The Jigsaw classroom. Beverly Hills: CA. Sage.

CASSANY, D. (2004). «Aprendizaje cooperativo para ELE». Actas del programa de formación para profesorado de español como lengua extranjera 2003-2004. Múnich: Instituto Cervantes.

Consejo De Europa (2002): Marco Común Europeo de Referencia para las Lenguas: aprendizaje, enseñanza, evaluación. Madrid: MECD y Anaya.

Instituto Cervantes (2006). Plan Curricular del Instituto Cervantes (PCIC). Madrid: Biblioteca Nueva.

JOHNSON Y JOHNSON (1999). El aprendizaje cooperativo en el aula. Barcelona: Editorial Paidós.

Kagan, S. (1994). Cooperative Learning. San Juan Capistrano: Kagan Cooperative Learning.

OVEJERo, A. (1990). El aprendizaje cooperativo. Una alternativa eficaz a la enseñanza tradicional. Barcelona: PPU.

Trosborg, A. (1995). Interlanguage pragmatics. Requests, complaints and apologies. Berlín y Nueva York: Mouton de Gruyter. 


\section{ANEXO:}

\section{Carta 1}

María Luisa González Gutiérrez

c/ Del Puerto, $32^{\circ} \mathrm{A}$

39080 Santander

Apartamentos Sol y Mar

C/ Piedras Blancas 4

29070 Málaga

Asunto: Reclamación por incumplimiento de características

Estimados señores:

Me pongo en contacto con ustedes para transmitirles mi insatisfacción por los servicios ofrecidos por su empresa.

Durante el puente de Semana Santa me alojé en uno de sus apartamentos. Hice la reserva porque había conexión wifi en el apartamento, así como minibar, vistas a la playa y acceso directo a la piscina. En el anuncio decía que la tranquilidad y el descanso estaban asegurados. Sin embargo, esos días comprobé que nada se acercaba a la realidad.

En primer lugar, la conexión wifi solo funcionaba en la recepción del hotel y en el jardín, lugares con mucho bullicio, lo que me impidió contestar los correos electrónicos de mis clientes durante mi estancia. En segundo lugar, me llevé una gran sorpresa al abrir la ventana, mi habitación daba a un callejón estrecho y oscuro donde estaba la entrada de la discoteca. Por otra parte, cuando intenté que me cambiaran de habitación, me dijeron en un tono poco agradable que no era posible porque el hotel estaba completo y nos habíamos registrado muy tarde. En cuanto al minibar, solo encontramos un botellín de agua en el apartamento. Para empeorar la situación, debíamos cruzar todas las instalaciones del hotel hasta llegar a una piscina minúscula.

Ante tales circunstancias, intenté reiteradamente ponerme en contacto con su empresa a través del teléfono para solucionar los inconvenientes surgidos pero, desgraciadamente, nadie me contestó.

Como prueba de todo lo expuesto, les adjunto las fotografías que tomé en el apartamento y en las instalaciones del complejo turístico.

Por todo ello, les reclamo una compensación por los daños ocasionados.

Espero recibir en breve una respuesta positiva para solucionar este problema. De lo contrario, me veré obligada a tomar medidas legales.

Atentamente,

María Luisa González Gutiérrez

Carta 2:

Susana Saiz Martínez

c/ Alfonso Jiménez, $206^{\circ} \mathrm{D}$ 
28070 Madrid

Airphone Telefonía, S.A.

Pol. Ind. Sierra Alta, 23

28071 Madrid

Madrid, 7 de octubre de 2015

Asunto: Error en la factura del teléfono

Muy señores míos:

Soy Susana Saiz Martínez y a través de esta carta me dirijo a ustedes con el objetivo de presentar una reclamación por los errores que aparecen en mi última factura de teléfono fijo de Airphone.

En la factura referencia de la que adjunto copia en esta carta aparecen llamadas y contrataciones de servicios que no he realizado y no son posibles porque durante la primera quincena de septiembre no ha residido nadie en mi domicilio, he estado de vacaciones en Nueva Zelanda. Durante un mes he explicado este problema al Servicio de Atención al cliente por teléfono, pero no me han dado ninguna solución, me decían que no había ningún error.

Por este motivo, le ruego que adopte las medidas oportunas para que se proceda a las verificaciones necesarias, tanto de las notas de facturación, como lo de los instrumentos de medida y demás comprobaciones técnicas. Espero que, de este modo, se compruebe que la facturación no es correcta y en consecuencia les requiero que la cantidad indebidamente facturada me sea compensada en la próxima factura.

Ante esta situación, les exijo una respuesta rápida. De lo contrario, me veré obligada a tomar medidas legales.

Atentamente,

Susana Saiz Martínez

Carta 3:

Sergio Santiago Ruiz

c/ Castillo Real, 76

48005 Bilbao

Líneas Aéreas VuelaRápido

Pol. Ind. Las Arenas, s/n

08007 Barcelona

Bilbao, 10 de febrero de 2016 
Asunto: Reclamación por cancelación de vuelo

Muy señores míos:

Me dirijo a ustedes con la intención de presentar una reclamación por la incidencia que sufrí el pasado día 6 de enero en el vuelvo $n^{o} 38901$ de su compañía, con origen Bilbao y destino Sevilla.

Ese día me presenté en el mostrador de su compañía para facturar mi maleta y nadie me informó de que el vuelo había sido cancelado. Justo en el momento de embarcar nos comunicaron al resto de pasajeros y a mí que el vuelo se cancelaba sin explicarnos el motivo, mientras otros vuelos despegaban sin problemas. Como consecuencia de este hecho, mi equipaje se extravió y me vi a obligada a comprar un billete de autobús para viajar y llegar a tiempo a mi destino.

Al no poder efectuar el viaje en condiciones de satisfacción, les pido que me devuelvan el importe del billete, así como una compensación económica por el equipaje extraviado y el billete de autobús que tuve que comprar.

Dejé constancia de esta incidencia en el servicio de Atención al Consumidor del aeropuerto el pasado día 7 de enero, pero a día de hoy y tras más de un mes, todavía estoy esperando que me den una respuesta. Quedo a la espera de sus noticias, de lo contrario, me veré obligado a tomar medidas legales.

Atentamente,

Sergio Santiago Ruiz 